\title{
Liprin- $\alpha 4$ as a New Therapeutic Target for SCLC as an Upstream Mediator of HIF1 $\alpha$
}

\author{
HIDEYA ONISHI ${ }^{1}$, AKIO YAMASAKI ${ }^{1}$, KATSUYA NAKAMURA $^{1}$, SHU ICHIMIYA ${ }^{1}$, \\ KOSUKE YANAI ${ }^{1}$, MASAYO UMEBAYASHI ${ }^{2}$, SHUNTARO NAGAI ${ }^{3}$ and TAKASHI MORISAKI ${ }^{2}$ \\ ${ }^{1}$ Department of Cancer Therapy and Research, Graduate School of Medical Sciences, \\ Kyushu University, Fukuoka, Japan; \\ ${ }^{2}$ Fukuoka General Cancer Clinic, Fukuoka, Japan; \\ ${ }^{3}$ Department of Surgery and Oncology, Graduate School of Medical Sciences, Kyushu University, Fukuoka, Japan
}

\begin{abstract}
Background/Aim: Small-cell lung cancer (SCLC) remains one of deadliest types of cancers. Cisdiamminedichloroplatinum (CDDP) is a key chemotherapeutic agent for SCLC, however, its therapeutic effect is limited. Recently, hypoxia in the cancer microenvironment has been suggested to influence the effect of cancer therapy. Materials and Methods: Using small interfering RNA inhibition of leukocyte common antigen-related interacting protein alpha 4

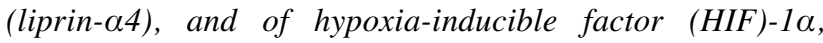
proliferation, invasion, migration and chemosensitivity were investigated in SBC-5 SCLC cells, under normoxia and hypoxia. Results: Liprin- $\alpha 4$ was found to contribute to proliferation, but not migration and invasion of SBC-5 cells both under normoxia and hypoxia. Inhibition of liprin- $\alpha 4$ increased chemosensitivity of SBC-5 cells under hypoxia. Liprin- $\alpha 4$ signaling occurs through mitogen-activated protein kinase pathways via activation of HIFla expression. Inhibition of HIFla reduced proliferation and increased chemosensitivity of SBC-5 cells under hypoxia. Conclusion: Liprin-a4 inhibition may enhance the effect of CDDP and liprin-a4 might be a novel therapeutic target in SCLC.
\end{abstract}

Small-cell lung cancer (SCLC) is still a refractory cancer for which there are few therapeutic strategies. Cisdiamminedichloroplatinum (CDDP) is a key chemotherapeutic agent, however, its effect is limited. Developing a new effective therapeutic strategy against SCLC and increasing the effect of existing agents is earnestly required.

Correspondence to: Hideya Onishi, Department of Cancer Therapy and Research, Graduate School of Medical Sciences, Kyushu University, 3-1-1 Maidashi, Higashi-ku, Fukuoka 812-8582, Japan. Tel: +81 926426220, Fax: +81 926426221, e-mail: ohnishi@surg1.med.kyushuu.ac.jp

Key Words: Liprin- $\alpha 4$, SCLC, HIF1 $\alpha$, invasion, proliferation.
The microenvironment surrounding cancer tissue is important in considering cancer therapy. This microenvironment includes tumor-infiltrating immune cells, fibrosis, and desmoplasia, as well as cytokines and other molecules. Hypoxia is also thought to occur in the cancer microenvironment. The tumor local site is extremely hypoxic, with an oxygen concentration of approximately $1 \% \mathrm{O}_{2}(1)$. Biological functioning is altered under hypoxia. Therefore, the cell phenotype should especially be considered when investigating proliferation, invasion, and chemosensitivity under hypoxia rather than under normoxia which is a usual experimental condition.

Liprins are major protein constituents of synapses and play a pivotal role in the organization of synaptic vesicles and neurotransmitter receptors (2). Liprin- $\alpha$ belongs to the LAR family of receptor protein tyrosine phosphatase $(3,4)$. Among liprin members, liprin-1 $\alpha$ is well researched and recently its interaction with some cancer types has been reported $(5,6)$. On the other hand, the role of liprin- $\alpha 4$ remains unclear with regard to cancer biology. Previously we showed that liprin- $\alpha 4$ has potential as a new therapeutic target for refractory pancreatic cancer (7). In the present study, we evaluated whether liprin- $\alpha 4$ might be a therapeutic target in SCLC, which like pancreatic cancer is a refractory type of neuroendocrine cancer.

\section{Materials and Methods}

Cell culture and reagents. A human SCLC cell line (SBC-5: American Type Culture Collection, Manassas, VA, USA) was maintained in RPMI-1640 medium (Nacalai Tesque, Kyoto, Japan) supplemented with $10 \%$ fetal calf serum (FCS; Life Technologies, Grand Island, NY, USA) and antibiotics (100 units/ml of penicillin and $100 \mu \mathrm{g} / \mathrm{ml}$ of streptomycin). Cell numbers were counted under light microscopy for cell proliferation assay.

For normoxic conditions, cells were cultured in $5 \% \mathrm{CO}_{2}$ and $95 \%$ air. For hypoxic conditions, cells were cultured in $1 \% \mathrm{O}_{2}, 5 \%$ $\mathrm{CO}_{2}$, and $94 \% \mathrm{~N}_{2}$ using a multigas incubator (Sanyo, Tokyo, Japan). 
RNA interference. Small interfering RNA (siRNA) for liprin- $\alpha 4$ (PTPRF interacting protein alpha 4, PPFIA4; ON-TARGET plus SMART pool, L-190474), hypoxia inducible factor-1 $\alpha$ (HIF1A; OnTARGET plus Smart pool, L-004018) and negative control siRNA (ON-TARGET plus si CONTROL non-targeting pool, D-001810) were purchased from Dharmacon RNA Technologies (Chicago, IL, USA). Cells $\left(2 \times 10^{5}\right.$ cells/well $)$ seeded in 6 -well plates were transfected with $100 \mathrm{nM}$ siRNA using Lipofectamine RNAiMAX Reagent (Thermo Fisher Scientific, Waltham, MA USA) according to the manufacturer's instructions. Cells were assayed at 2 days after transfection.

Matrigel invasion assay and migration assay. The invasiveness of SBC-5 cells was assessed based on the invasion of cells through Matrigel-coated transwell inserts as described previously (8). In brief, the upper surface of a filter (pore size, $8.0 \mu \mathrm{m}$; BD Biosciences, Heidelberg, Germany) was coated with basement membrane Matrigel (BD Biosciences). After cells were transfected with control siRNA or liprin- $\alpha 4$ siRNA for 2 days, $8 \times 10^{4}$ cells were added to the upper chamber and incubated for $16 \mathrm{~h}$ both under normoxia and hypoxia. After incubation, the filter was fixed and stained with Diff-Quick reagent (International Reagents, Kobe, Japan). All cells that had migrated from the upper to the lower side of the filter were counted under a light microscope (BX50; Olympus, Tokyo, Japan) at a magnification of $\times 100$. Tumor cell invasiveness testing was carried out in triplicate wells.

Cell migration assay was performed in the same way using Matrigel-non-coated transwell inserts.

Chemosensitivity. After cells were transfected with control siRNA, liprin- $\alpha 4$ siRNA or HIF1a siRNA for 2 days, cells were plated in 24 -well plate at the cell density of $0.1 \times 10^{6}$ cells/well. Cells were then treated for 2 days with 5-fluorouracil (5-FU, $100 \mu \mathrm{g} / \mathrm{ml}$; Bristol Myers Squibb, New York, NY, USA) or CDDP (50, 500, $600,1,000$ or $1,200 \mu \mathrm{g} / \mathrm{ml}$; Kyowahakkou, Tokyo, Japan) under both normoxia and hypoxia. Viable cancer cells were subsequently counted under light microscopy and chemosensitivity was calculated as a ratio of the number of viable cancer cells to the number of viable control cells.

Western blotting. After cells were transfected with control siRNA, liprin- $\alpha 4$ siRNA or HIF1a siRNA for 2 days, cells were cultured under both normoxia and hypoxia for 1 day. Whole cell extraction was then performed with M-PER Reagents (Pierce Biotechnology, Rockford, IL, USA) according to the manufacturer's instructions. Protein samples $(50 \mu \mathrm{g})$ were separated by electrophoresis on a sodium dodecyl sulfate-polyacrylamide gel and transferred to Protran nitrocellulose membranes (Whatman GmbH, Dassel, Germany). The protein-bearing membranes were incubated overnight at $4^{\circ} \mathrm{C}$ with primary antibodies for HIF1 $\alpha$ (1:100; Santa Cruz Biotechnology, Santa Cruz, CA, USA), liprin- $\alpha 4$ (1:200; Abnova, Walnut, CA, USA), phospho-extracellular signal-regulated kinase (ERK)1/2 (1:200; Cell Signaling Technology, Danvers, MA, USA), ERK1/2 (1:200; Cell Signaling Technology), phospho(p)AKT serine/threonine kinase (AKT1/2/3, 1:200; Santa Cruz Biotechnology), and AKT1/2/3 (1:200; Santa Cruz Biotechnology). Peroxidase-linked secondary antibodies (Amersham Biosciences, Piscataway, NJ, USA) were subsequently added and the membranes were further incubated for $1 \mathrm{~h}$ at room temperature. Antibody for $\alpha$-tubulin (1:1,000; Sigma-Aldich, St. Louis, MO, USA) was used as protein loading control.
Statistical analysis. The data are presented as the means \pm standard deviation (SD). Student's $t$-tests were used to compare continuous variables between two groups. Differences with $p$-values of less than 0.05 were considered as statistically significant.

\section{Results}

Liprin- $\alpha 4$ contributes to proliferation of $S B C-5$ cells. Firstly, we analyzed whether liprin- $\alpha 4$ is involved in biological functions in SBC-5 cells. The number of liprin- $\alpha 4$ siRNAtransfected cells after 3-day incubation was significantly lower than that of control cells under both normoxia and hypoxia (Figure 1A). However, inhibition of liprin- $\alpha 4$ did not lead to a significant difference in migrating or invading cell numbers compared to those of control-transfected cells, while cell numbers under hypoxia were significantly lower than those under normoxia (Figure $1 \mathrm{~B}$ and $\mathrm{C}$ ). These results suggest that liprin- $\alpha 4$ contributes to proliferation of SBC-5 cells, but not to their migration or invasion under normoxia and hypoxia.

Inhibition of liprin- $\alpha 4$ increased chemosensitivity of SBC-5 cells under hypoxia. Combinational use of chemotherapeutic and molecular targeting drugs is an important strategy for therapy of refractory cancer. Therefore, chemosensitivity of combinational use of CDDP or 5-FU with liprin- $\alpha 4$ inhibition was evaluated under normoxia and hypoxia. The concentrations of CDDP and 5-FU used were determined by previous experiments (9). Chemosensitivity to CDDP and 5FU was dramatically reduced under hypoxia compared to that under normoxia (Figure 2A). Liprin- $\alpha 4$ inhibition significantly increased chemosensitivity to CDDP under hypoxia but not under normoxia (Figure 2A). Next, whether this increased chemosensitivity to CDDP was dependent on CDDP concentration was evaluated. Unfortunately, increased chemosensitivity to CDDP was found only at relatively high concentration, $1000 \mu \mathrm{g} / \mathrm{ml}$ under hypoxia, while chemosensi-tivity to CDDP under hypoxia was reduced compared to that under normoxia at all CDDP concentrations tested (Figure 2B).

Liprin-a4 signaling is through MAPK pathways via the activation of HIFl $\alpha$ expression. HIF $1 \alpha$ is an important transcriptional factor under hypoxia. We investigated the relation between liprin- $\alpha 4$ and HIF $1 \alpha$. The expression of liprin- $\alpha 4$ was increased under hypoxia compared to that under normoxia (Figure 3A). Interestingly, inhibition of liprin- $\alpha 4$ reduced the expression of HIF1 $\alpha$ under hypoxia (Figure $3 \mathrm{~B}$ ). On the other hand, HIF1 $\alpha$ inhibition did not appear to affect the expression of liprin- $\alpha 4$ under hypoxia (Figure 3C). In previous study, we showed that liprin- $\alpha 4$ is involved in the activation of phosphoinositide 3-kinase (PI3K) and mitogenactivated protein kinase (MAPK) signaling pathways in pancreatic cancer under hypoxia (7). Therefore, next, we 
A

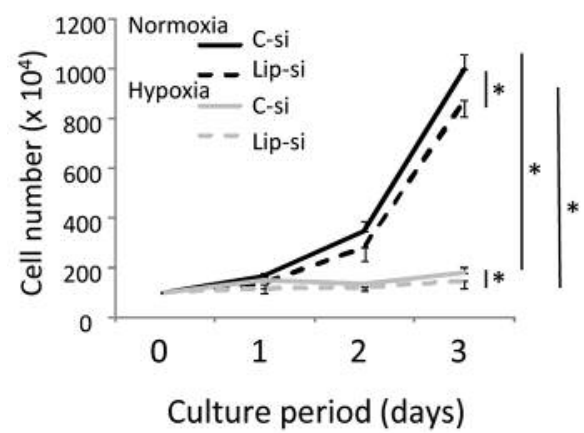

B

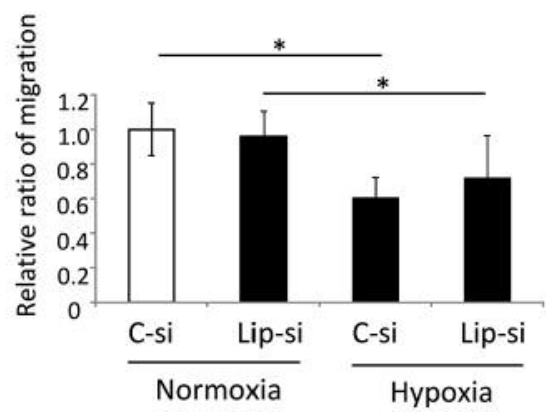

C

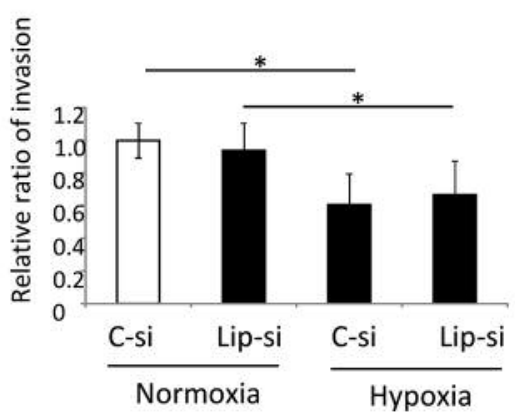

Figure 1. Liprin- $\alpha 4$ contributes to proliferation of SBC-5 cells. A: Cell proliferation was analyzed under both normoxia and hypoxia. Cell numbers at the indicated days in SBC-5 cells transfected with liprin- $\alpha 4$ siRNA (Lip-si) were counted under light microscopy and compared with cells transfected with control siRNA $(C$-si). Cell migration $(B)$ and invasiveness $(C)$ were estimated by Matrigel invasion assay. All cells that had migrated from the upper to the lower side of the filter were counted. Data are presented as means \pm standard deviation. *Significantly different at $p<0.05$.

A

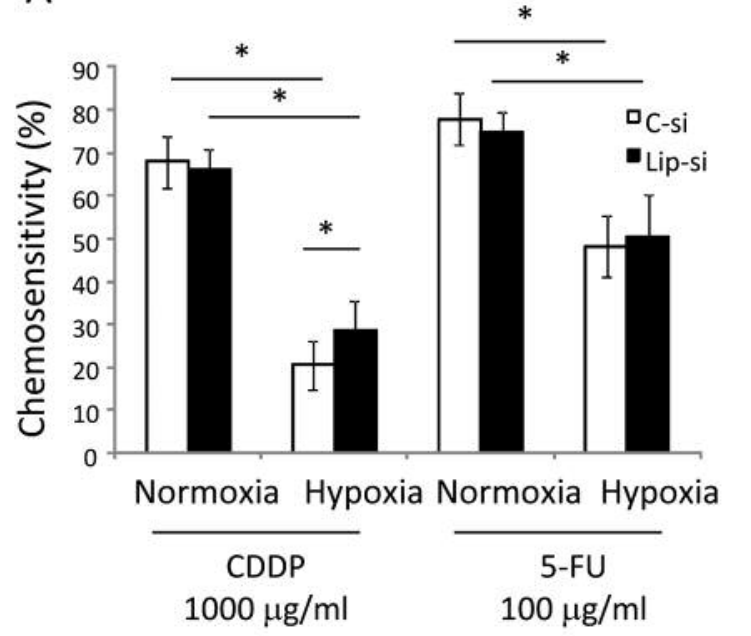

B

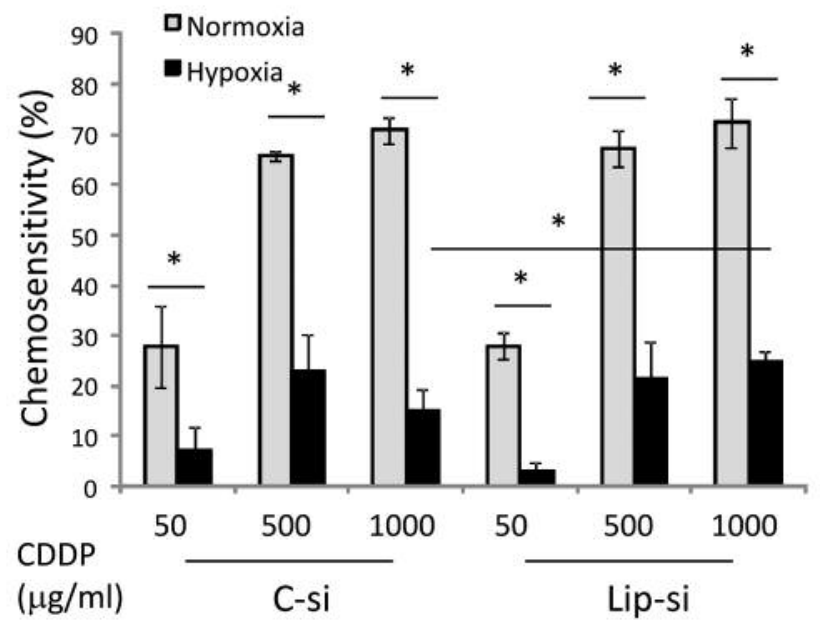

Figure 2. Inhibition of liprin- $\alpha 4$ increased chemosensitivity of SBC-5 cells under hypoxia. Liprin- $\alpha 4$ siRNA-transfected (Lip-si), and control siRNAtransfected (C-si) SBC-5 cells were used. Viable SBC-5 cell numbers after 2 days of treatment with 5-fluorouracil (5-FU) and cisdiamminedichloroplatinum $(C D D P)$ at the indicated concentration $(A)$ and with CDDP at different concentrations $(B)$ under normoxia and hypoxia were counted using light microscopy. Chemosensitivity was calculated as a ratio of the number of viable cancer cells to the number of viable control cells. Bars indicate the standard deviation. *Significantly different at $p<0.05$.

investigated whether liprin- $\alpha 4$ contributes to the activation of PI3K and MAPK pathways under hypoxia in SBC-5 cells. Activation of ERK (pERK) reduced when liprin- $\alpha 4$ in SBC-5 cells was inhibited, while there was no significant difference in pAKT level (Figure 3D). Under HIF1 $\alpha$ inhibition, pERK was practically abolished. These results suggest that under hypoxia liprin- $\alpha 4$ signaling in SBC-5 cells occurs through MAPK pathways via HIF1 $\alpha$ expression.
Inhibition of HIFla reduced proliferation and increased chemosensitivity of SBC-5 cells under hypoxia. Next, we investigated whether HIF $1 \alpha$ contributes to proliferation and chemosensitivity of SBC-5 cells. Inhibition of HIF1 $\alpha$ significantly reduced proliferation of SBC-5 cells under hypoxia (Figure 4A). Inhibition of HIF1 $\alpha$ also significantly enhanced chemosensitivity of SBC-5 cells under hypoxia (Figure 4B). 


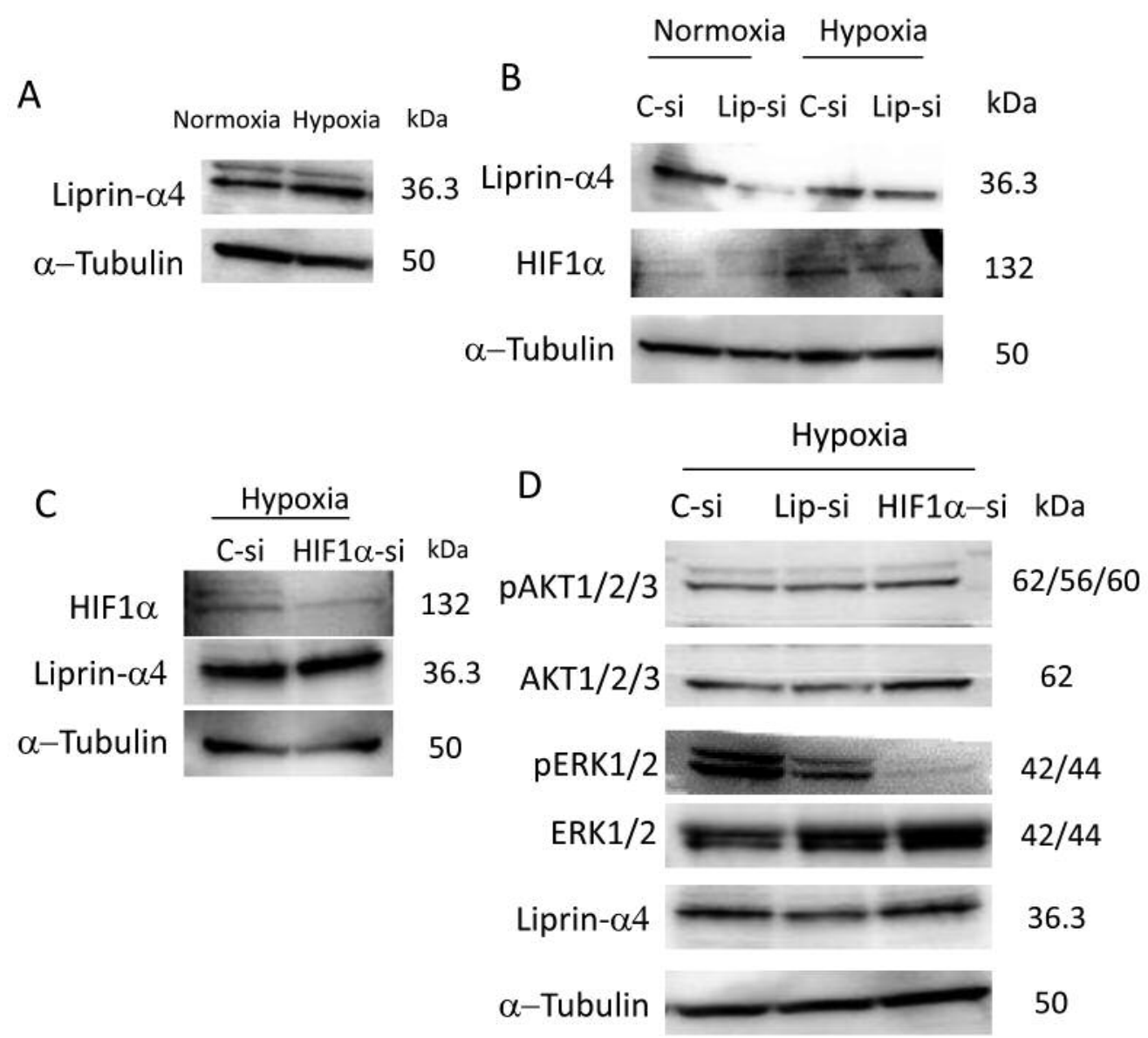

Figure 3. Liprin- $\alpha 4$ regulates expression of hypoxia-inducible factor-1 $\alpha$ (HIF $1 \alpha)$. A: After SBC-5 cells were incubated under normoxia and hypoxia for $24 \mathrm{~h}$, whole protein was extracted and $50 \mu \mathrm{g}$ of protein was applied for western blotting. B, C and D: Liprin- $\alpha 4$ siRNA (Lip-si), HIF1 $\alpha$ siRNA and control siRNA (C-si) were transfected with SBC-5 cells for 2 days under normoxia. SBC-5 cells were then incubated under normoxia and hypoxia for an additional $24 \mathrm{~h}$. Protein expression of liprin- $\alpha 4$, HIFl $\alpha$, phospho-AKT serine/threonine kinase (pAKT), AKT, extracellular signalregulated kinase (ERK) and pERK were investigated in western blotting using $50 \mu \mathrm{g}$ of protein. $\alpha$-Tubulin was used as a loading control.

\section{Discussion}

Figure 4C depicts our basic study findings. Hypoxia appears to up-regulate the expression of liprin- $\alpha 4$, which induces the expression of HIF1 $\alpha$. HIF1 $\alpha$ contributes to increased proliferation and lower chemosensitivity. Therefore, inhibition of liprin- $\alpha 4$ or HIF $1 \alpha$ led to reduced proliferation and increased chemosensitivity of SBC-5 cells.

Hypoxia is an important feature of the tumor microenvironment. We analyzed gene expression under hypoxia in pancreatic cancer and found liprin- $\alpha 4$ to be increased (7). That inhibition of liprin- $\alpha 4$ reduced proliferation and invasiveness in pancreatic cancer was shown in our previous work (7). In the present study using an SCLC line, SBC-5, the inhibition of liprin- $\alpha 4$ reduced proliferation, but not migration and invasiveness. Among liprin proteins, liprin- $\alpha 1$ is well researched and it was shown that liprin- $\alpha 1$ is involved in the invasiveness of cancer cells $(5,6)$. But it is a matter of course that each liprin family should play a different role according to the type of cancer. Investigating many kinds of cancers is needed in order to be able to develop a comprehensive cancer therapy targeting liprin- $\alpha 4$.

Because chemotherapy agents target cells undergoing frequent nuclear mitosis, it would be expected that if proliferation decreases, chemosensitivity should decrease. But our result using liprin- $\alpha 4$ inhibition of cells was entirely opposite to this. In addition, chemosensitivity of SBC-5 cells was dramatically reduced under hypoxia. One explanation for this may be the decrease of HIF1 $\alpha$ expression due to liprin- $\alpha 4$ inhibition. HIF $1 \alpha$ is an important transcriptional factor under hypoxia. It has been reported that HIF1 $\alpha$ 

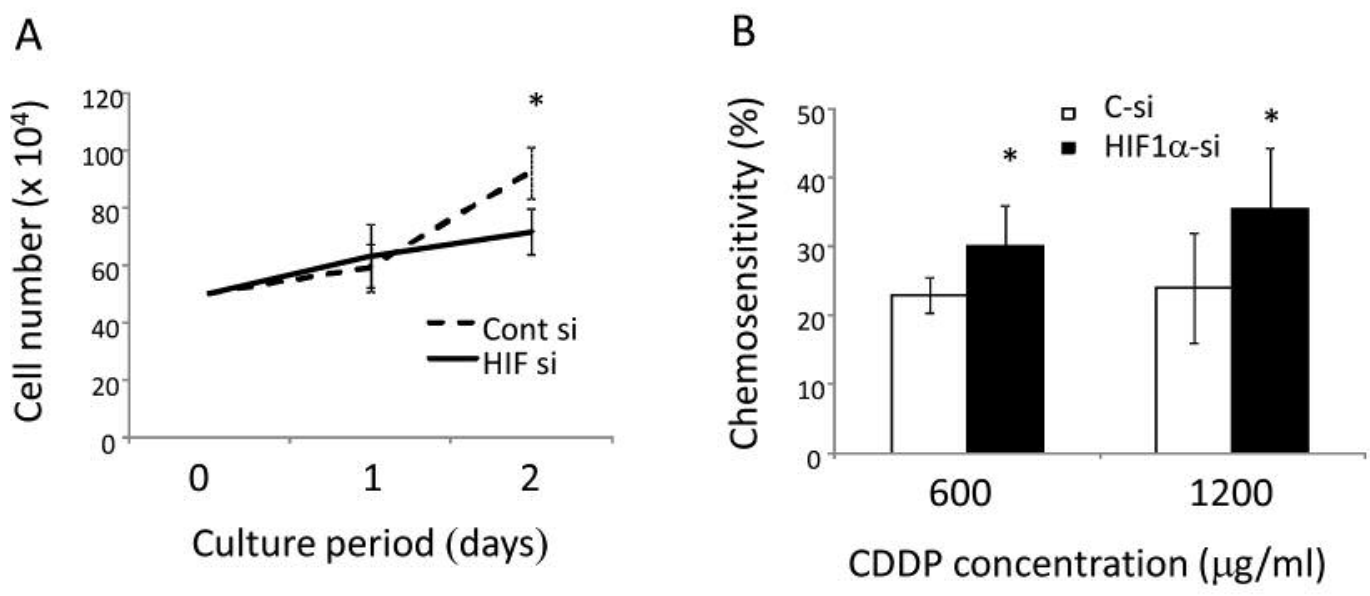

C

SBC-5 cells

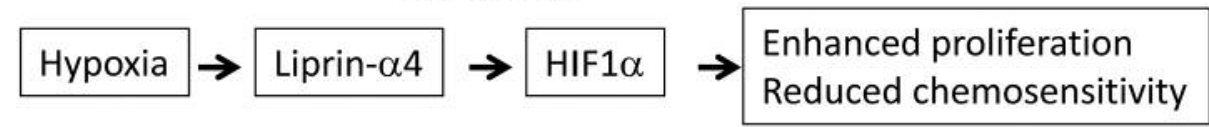

Figure 4. Inhibition of hypoxia-inducible factor-1 $\alpha$ (HIF1 $\alpha)$ reduced proliferation and increased chemosensitivity of SBC-5 cells under hypoxia. A: Cell proliferation of SBC-5 cells transfected with HIF1a siRNA was analyzed under hypoxia. Cell numbers at the indicated days were counted under light microscopy. B: HIFIa siRNA-transfected SBC-5 cells and control siRNA-transfected (C-si) SBC-5 cells were used. Viable SBC-5 cell numbers after 2 days of treatment under hypoxia with cis-diamminedichloroplatinum (CDDP) at the indicated concentration were counted using light microscopy. Chemosensitivity was then calculated as the ratio of the number of viable cancer cells to the number of viable control cells. Bars indicate standard deviation. *Significantly different at $p<0.05$. C: Schematic figure is indicated. Hypoxia up-regulates the expression of liprin- $\alpha 4$, which induces the expression of HIF $1 \alpha$. HIF $1 \alpha$ contributes to increased proliferation and reduced chemosensitivity.

promotes chemoresistant tumor cells (10). Considering these results, the inhibition of liprin- $\alpha 4$ is thought to be a good therapeutic strategy.

The relation between liprin- $\alpha 4$ and HIF $1 \alpha$ is controversial. It was shown that liprin- $\alpha 4$ is directly regulated by HIF1 $\alpha$ in renal cell carcinoma (11). However, our result that HIF1 $\alpha$ is regulated by liprin- $\alpha 4$ is thoroughly opposite. Although signal transduction may be different according to the type of cancer, this should be confirmed in another SCLC line.

Our results suggest that findings in conventional experiments under normoxia may not reflect in vivo results. With regard to this, the result that the inhibition of liprin- $\alpha 4$ improved chemosensitivity to CDDP under hypoxia is a promising observation. Our results strongly imply that liprin$\alpha 4$ inhibition may augment the effect of CDDP and liprin$\alpha 4$ might be a good therapeutic target for SCLC.

\section{Conflicts of Interest}

The Authors declare no financial or commercial conflict of interest in regard to this study.

\section{Authors' Contributions}

Onishi contributed all experiments analyzed. Yamasaki contributed western blotting. Ichimiya contributed gene transfection. Umebayashi and Morisaki contributed interpretation of data. Nagai contributed acquisition and analysis of data. Nakamura and Yanai contributed design of the work.

\section{Acknowledgements}

The Authors thank Ms. Emi Onishi for skillful technical assistance. This study was supported by JSPS KAKENHI Grant Number 17H04283, 18K08620 and 18K08788.

\section{References}

1 Hockel S, Schlenger K, Vaupel P and Hockel M: Association between host tissue vascularity and the prognostically relevant tumor vascularity in human cervical cancer. Int J Oncol 19: 827832, 2001. PMID: 11562762.

2 Spangler SA, Jaarsma D, De Graaff E, Wulf PS, Akhmanova A and Hoogenraad CC: Differential expression of liprin- $\alpha$ family proteins in the brain suggests functional diversification. J Comp Neurol 519: 3040-3060, 2011. PMID:21618222. DOI: 10.1002/ cne.22665. 
3 Serra-Pagès C, Medley QG, Tang M, Hart A and Streuli M: Liprins, a family of LAR transmembrane protein-tyrosine phosphatase-interacting proteins. J Biol Chem 273: 1561115620, 1998. PMID: 9624153.

4 Serra-Pagès C, Kedersha NL, Fazikas L, Medley Q, Debant A and Streuli M: The LAR transmembrane protein tyrosine phosphatase and a coiled-coil LAR-interacting protein colocalize at focal adhesions. EMBO J 14: 2827-2838, 1995. PMID: 7796809.

5 Astro V, Asperti C, Cangi MG, Doglioni C and de Curtis I: Liprin- $\alpha 1$ regulates breast cancer cell invasion by affecting cell motility, invadopodia and extracellular matrix degradation. Oncogene 30: 1841-1849, 2011. PMID: 21151172. DOI: 10.1038/onc.2010.562.

6 Pehkonen H, von Nandelstadh P, Karhemo PR, Lepikhova T, Grenman R, Lehti $\mathrm{K}$ and Monni O: Liprin- $\alpha 1$ is a regulator of vimentin intermediate filament network in the cancer cell adhesion machinery. Sci Rep 14: 24486, 2016. PMID: 27075696. DOI: $10.1038 /$ srep24486.

7 Yamasaki A, Nakayama K, Imaizumi A, Kawamoto M, Fujimura A, Oyama Y, Nagai S, Yanai K and Onishi H: Liprin- $\alpha 4$ as a possible new therapeutic target for pancreatic cancer. Anticancer Res 37: 6649-6654, 2017. PMID: 29187440. DOI: 10.21873/ anticanres.12122.
8 Onishi H, Nakamura K, Nagai S, Yanai K, Yamasaki A, Kawamoto M, Imaizumi A and Morisaki T: Hedgehog inhibition upregulates TRK expression to antagonize tumor suppression in small cell lung cancer cells. Anticancer Res 37: 4987-4992, 2017. PMID: 28870922. DOI: 10.21873/anticanres.11910.

9 Onishi H, Morifuji Y, Kai M, Suyama K, Iwasaki H and Katano M: Hedgehog inhibitor decreases chemosensitivity to 5fluorouracil and gemcitabine under hypoxic conditions in pancreatic cancer. Cancer Sci 103: 1272-1279, 2012. PMID: 22486854. DOI: 10.1111/j.1349-7006.2012.02297.x.

10 Xia Y, Jiang L and Zhong T: The role of HIF1 $\alpha$ in chemo/radioresistant tumors. Onco Targets Ther 11: 3003-3011, 2018. PMID: 29872312. DOI: 10.2147/OTT.S158206.

11 Mattauch S, Sachs M and Behrens J: Liprin- $\alpha 4$ is a new hypoxia-inducible target gene required for maintenance of cellcell contacts. Exp Cell Res 316: 2883-2392, 2010. PMID: 20599943. DOI: 10.1016/j.yexcr.2010.06.022.

Received January 3, 2019

Revised February 1, 2019

Accepted February 4, 2019 